\title{
Inhomogeneity of deformed state during compression testing of titanium implant
}

\author{
Yury Loginov ${ }^{1}$, Stepan Stepanov ${ }^{1, *}$, and Catherine Khanykova ${ }^{1}$ \\ ${ }^{1}$ Ural Federal University, 620002, 19 Mira str., Ekaterinburg, Russia
}

\begin{abstract}
This study examines the inhomogeneity of deformed state during compression testing of porous titanium implant. The theoretical part of the article includes numerical simulation of deformation of a prismatic titanium sample compressed with absolutely rigid plates. The porosity was provided by the circular shape pores with titanium struts between them. To solve the problem by means of the finite element analysis, the boundary conditions were set using the ABAQUS software. The fields of strain, stresses and displacements were determined. Presumable place of fracture coincide with the highest values of strain which are localized in vertical struts. Physical modeling of the implant compression was performed at the second part of the study on the testing machine with video recording. It was shown that in the real process the localization of failure corresponds to the calculated data.
\end{abstract}

\section{Introduction}

The implant industry takes an ever-increasing living space in the field of bioengineering. It's achievements allow for prolonging an active life of a human as well as "repairing" individual body parts [1]. Titanium alloys are often utilized as a material for such invasions due to their chemical inertness and high strength to weight ratio [2-4]. Manufacturing of titanium implants employs methods of powder metallurgy used to obtain the desired architecture (the ratio of the shape and the size of pores in materials) $[5,6]$. The predetermined porosity reduces the weight of implant, which allows for achieving the weight of natural bone tissue. A greater shock absorbing of joints, when impact and static loads take place, is provided by a smaller modulus of elasticity. The better intergrowth of the living tissues through pore space is ensured, thus integration with this organism is achieved.

Originally, porous structures of metals were obtained using the powder sintering. Sintering mode provided the required porosity. However, this did not allow for designing the architecture of the material at each point. The development of additive manufacturing enabled to define the structure of the material at macro level by using the software for controlling the process of a metal medium growing [7]. At the same time, the structural and phase state of the alloys used can be set $[8,9]$.

Although the modulus of elasticity of widely used titanium alloys can be adjusted by changing phase composition $[10,11,12]$, this method regulates the elastic modulus in a very limited range. A more effective technique is to change the porosity of the material [13]. In addition, the effect of the porous space architecture can be added. Thus, a whole class of so-called cellular materials origination appeared [14].
An additional elastic effect can be provided by a gas located within the pores due to the appearance of throttling effect through the pores with open porosity and compressive resistance effect for closed porosity [16]. The architecture of the volume of a porous medium is also significant. For example, the tortuosity of pores is introduced to account for the resistance to throttling in the mechanics of liquids and gases transiting in the porous media. Another lever for controlling the properties of porous materials is to modify the shape of the pores by methods of metal forming [16, 17], including isostatic treatment in a hydrostatic or gas-static version.

The compression testing of porous implants has some peculiarities, since their elastic and strength characteristics are determined both by the properties of the material from which they were made and by the shape, as well as by the size and arrangement of the pores.

The purpose of the work is to study the behavior of the material in the compression test of additive manufactured implant.

\section{Simulation}

This study employs mathematical modeling based on the finite element analysis (FEA) to describe a porous/cellular material with the simple circular cells uniformly distributed in the metallic medium.

A planar case is considered to simplify the stressstrain state. A schematic representation of such a structure is shown in Fig. 1. The architecture of the material in the plane is shown. It consists of the metal 1 and the circular pores 2 formed therein. The selected fragment 3 was utilized for calculations introduces the radius of curvature $R$ (as half the pore diameter) and the

\footnotetext{
* Corresponding author: s.i.stepanov@urfu.ru
} 
thickness of the strut $\delta$. In general, such a construction is regarded as a unit cell. The implant consists of a number of such cells. FEA simulation of the compression loading was performed using the ABAQUS software with the number of finite elements not exceeding 1000. The properties of the Ti-6Al-4V most frequently used alloy for implant production were set. It is characterized by the high strength and reasonable ductility. The strength properties are given by the dependence of the stress plastic flow $\sigma_{s}$ on the relative reduction $\varepsilon_{\%}$, expressed in percent. It is approximated by the formula $\sigma_{s}=a+b$ * $\varepsilon_{\%}{ }^{c}$, where $a=980 \mathrm{MPa} ; b=10.78 \mathrm{MPa}$; $c=0.76$ - empirical coefficients.

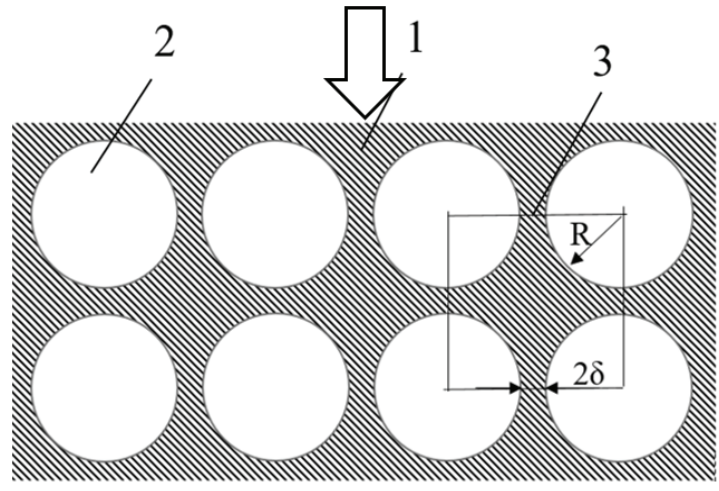

Fig. 1. Planar architecture of the porous titanium sample: 1 - metal; 2 - pore; 3 -a segment for simulation characterized by the radius $\mathrm{R}$ and the strut width $\delta$.

The configuration of the unit cell for calculations is shown in Fig. 2 a. The load is applied in the XY plane in the vertical direction along the $\mathrm{Y}$ axis. The contour of the deformed body consists of five segments $A, B, C, D, E$. The $\mathrm{Y}$ axis is considered as an axis of vertical symmetry; therefore, on the boundary $E$, the horizontal displacements $u_{y}$ on this axis are equal to zero. The $\mathrm{X}$ axis is considered as a horizontal axis of symmetry, therefore the vertical displacements $u_{y}$ are also equal to zero on the boundary $A$. At the boundary $D$, the vertical displacement transmitted by the deformation tool is specified. The boundary $C$ is free, thus no displacements take place there.

$D$

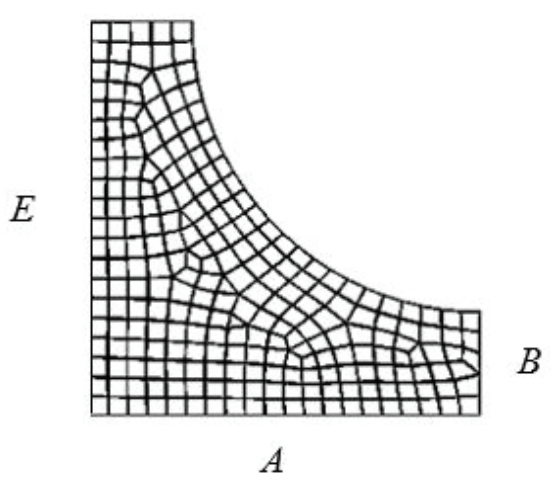

a)

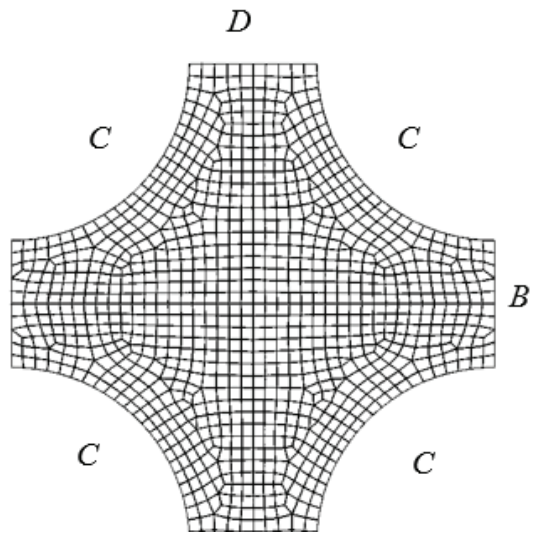

b)

Fig. 2. Boundary conditions in the graphic form (a) and the configuration of the deformation zone non-loaded state with allowance for the symmetry conditions (b).

In the basic solution, the ratio $R / \delta=75 / 25=3$ was adopted. The cell height was assumed equal to the $H_{0}=R+\delta$, then the relative reduction was $u_{y} / H_{0}$. The area of the square occupied by the unit cell is $H_{0} * H_{0}$, the pore area in this square is $\pi R^{2} / 4$, then the porosity can be estimated as the ratio of the area of the pore to the area of the square, expressed as a percentage: $P=100^{*} \pi R^{2} / 4 /(R+\delta)^{2}$. For the relations used in the study and for the flat formulation of the problem, we obtain the porosity $P=44 \%$.

\section{Simulation results and discussion}

Fig. 3 shows the vertical and horizontal displacement fields $U 2$ and $U 1$, respectively. The largest vertical displacement U2 (Fig. 3a) are localized near the contact surface. Displacements along the central vertical are higher than in the periphery. The horizontal displacements U1 have an inverse order of distribution: the largest in the periphery and the smallest in the center.

The distribution of equivalent plastic strain PEEQ and of the intensity of stresses $S$ for $u_{y} / H_{0}=3 /(7,5+2,5 / 2)=0,34$ is presented in Fig. 4 .

A field of equivalent plastic strain PEEQ in the scalar form is distributed unevenly in volume (Fig. 3).

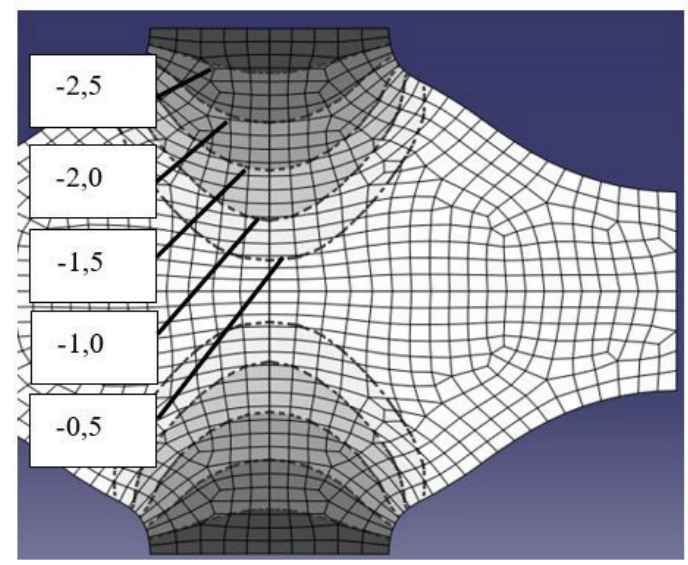

a) 


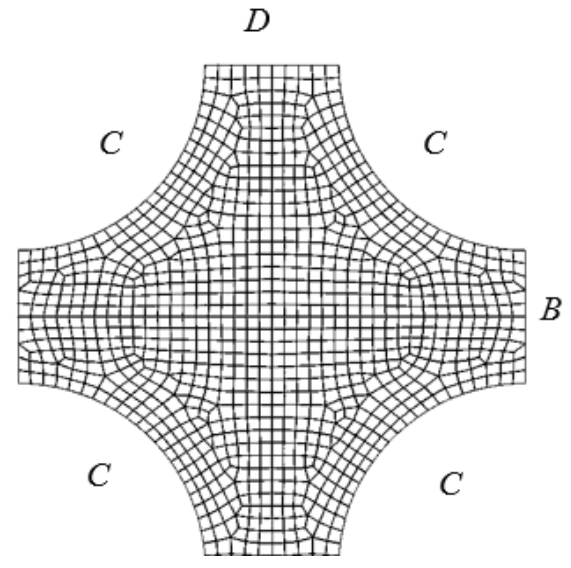

b)

Fig. 3. Field of vertical U2 (a) and horizontal U1 (b) displacements $(\mathrm{mm})$

A larger value of PEEQ is acquired for the metal layers located on the free surface near the contact with the deformation tool and in the center of the unit cell. In addition, the point of maximum deformation is displaced from the center of the strut by a half of the thickness. The stress intensity is localized at the same point. In general, the stress intensity decreases from the center to the periphery. This is explained with the increase in the cross-section area through which the load is transmitted. Note, that both equivalent stresses and deformations are maximal in vertical struts.
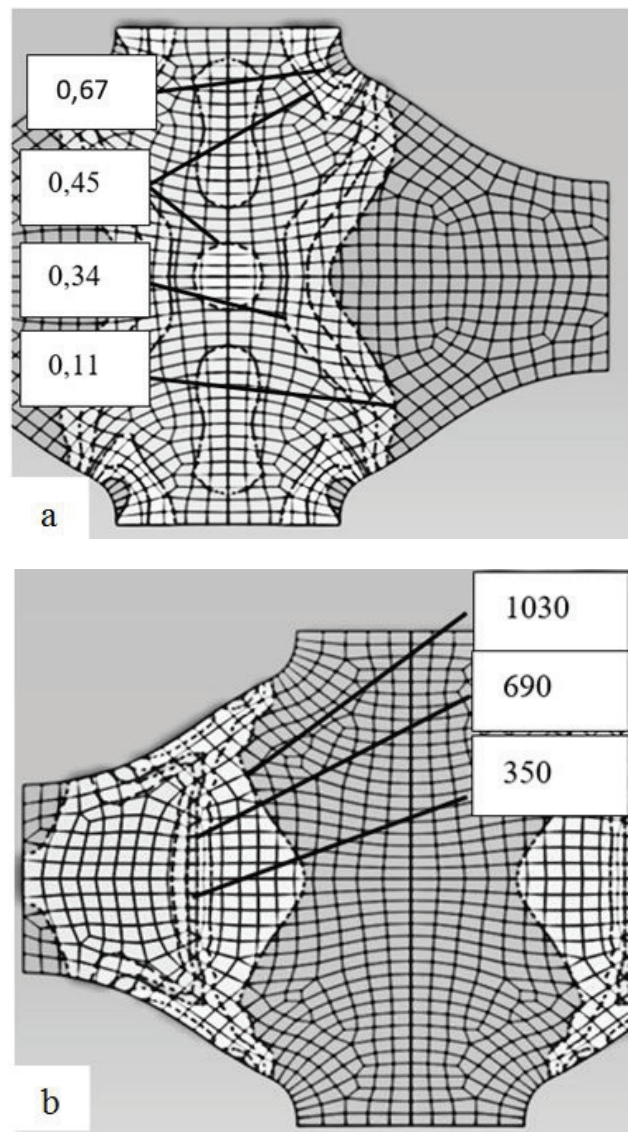

Fig. 4. Distribution of equivalent plastic strain PEEQ (a) and of the intensity of stress $\mathrm{S}(\mathrm{b})$ through the volume of the unit cell
Thus, the most loaded points were in the same place both for the deformed and for the stressed state. To assess the probability of failure, it is necessary to analyze the distribution of the mean (hydrostatic stresses), as shown in Fig. 5.

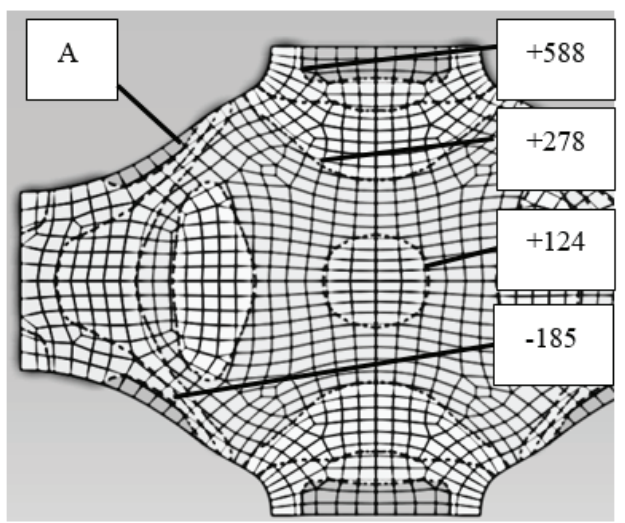

Fig. 5. Distribution of the pressure (mean stress taken with the opposite sign, $[\mathrm{MPa}]$ ) through the volume of the unit cell

The cell volume is characterized by the occurrence of both compressive and tensile mean stresses as can be seen from the Fig. 5. The average stresses are close to zero in the center of the cell and in the periphery. A high compressive stresses act on the upper boundary, which is explained with the very type of the applied load. The mean stress decreases from the center to the periphery by analogy with the stress intensity (in absolute value). The appearance of a zone of tensile stresses on a segment of the cell radius (designated as region $\mathrm{A}$ ) is of special interest.

Note, that the solution is based on the hypothesis that the material remains in a plastic state and does not become brittle. If it turns out that the ductility of the material decrease, then, instead of reaching the level of large plastic deformations, a brittle fracture will occur.

The simulation of the distribution of the stress-strain state parameters allows for estimation of the dangerous sections from the point of fracture of the whole structure. Such places in this case are the areas with the increased value of strain that are the horizontal bridges between the cells. In addition, the regions with tensile stresses appear near the vertical struts which can also lead to cracks formation and further fraction of the material. A general recommendation to improve strength of the total structure is to increase the thickness of the struts. At the same time, it should be remembered that the modulus of elasticity of the system will simultaneously increase.

\section{Experimental procedures}

The implant of the titanium alloy was manufactured by direct metal laser sintering using EOSINT 280 with a $200 \mathrm{~W}$ pulse laser power. The powder of the $(\alpha+\beta)-$ titanium alloy Ti-6Al-4V was utilized, the composition and properties of which correspond to ISO 5832-3-2014 for metallic implants for surgery. 


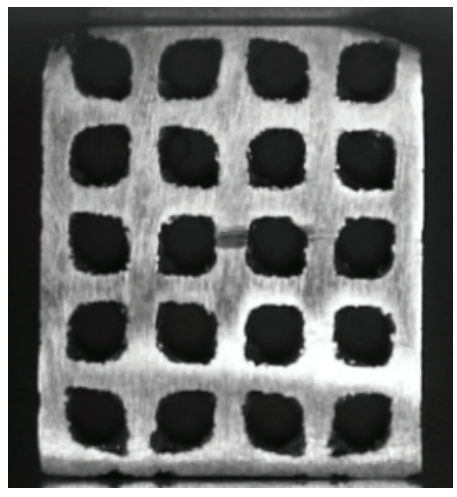

a)

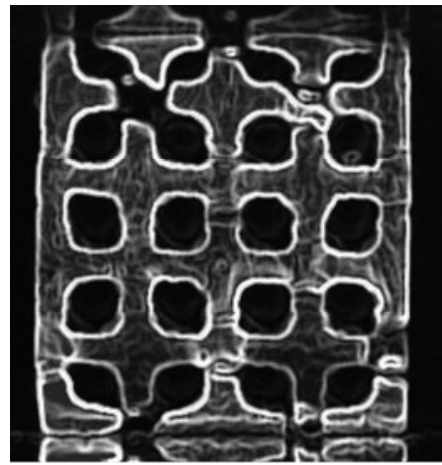

d)

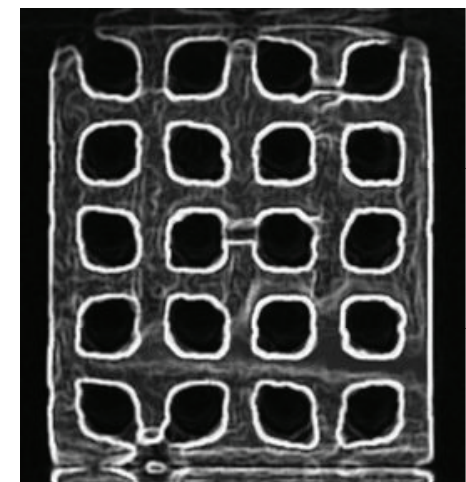

b)

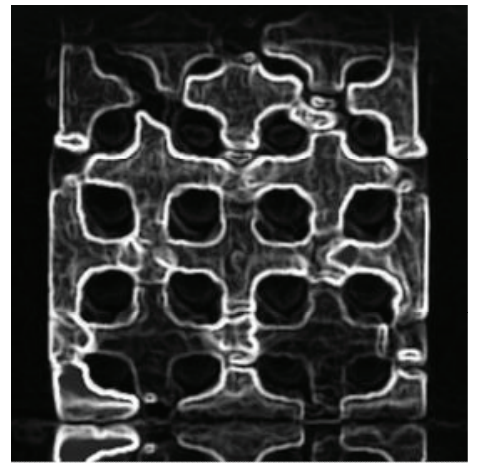

e)

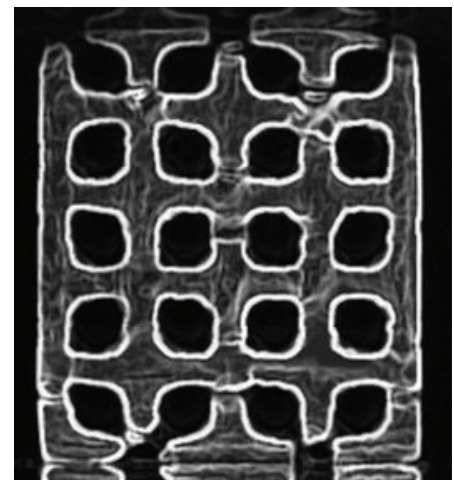

c)

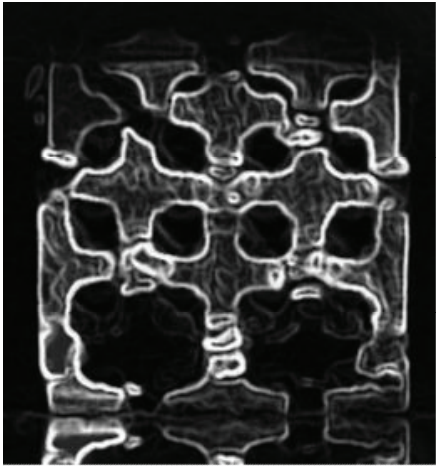

f)

Fig. 6. Cell/pore configuration changing for strains of (\%): 0(a); 0,7(b); 3,5(c); 9,2(d); 14,8(e); 27,1(f) during compression test

ISO 13314 specifies a test method for compressive properties of porous and cellular metals.

Rectangular prism shape samples with the width of $9.2 \mathrm{~mm}$, height of $11 \mathrm{~mm}$ and the length of $11,02 \mathrm{~mm}$ were sectioned from the implants. The samples were tested in compression using an Instron 3380 tensile testing machine at a traverse speed of $1.8 \mathrm{~mm} / \mathrm{min}$. Video recording was performed during compression testing along with the logging of strain and loading by the monitoring system of the testing machine.

\section{Results and discussion}

When the specimen is compressed (Fig. 1), the shape of circular pores in the cross section becomes elliptical, elongated in the orthogonal direction with respect to the compression direction $[18,19]$. However, such a scenario is optional, since the pore contour can provide additional resistance to deformation. What is obvious is that the $\mathrm{B}$ size (the width of the sample) under the compression would increase for an incompressible medium, but this is not obvious for a sample with pores.

A number of films typical of specific compression stages and corresponding to a certain relative strain (expressed in percent) were excerpted from the video record. The strain value depends on the method of measurement. If the sample were to be extracted every time to estimate this value, then the plastic component of the parameter would be calculated. But in this case the measurements were carried out immediately under load, so the represented relative reduction accounts for the sum of both the elastic and the plastic strain components. This sample collection of frames is shown in Fig. 6. All the drawings, except the first one, were modified by applying the function of increasing the contrast of the cell boundaries. This function provides a better distinguishing of the contours of these boundaries

The initial pore configuration is not a circumferential, since the secant plane did not intersect the channels at an expected angle (Fig. 6a) when sectioning the samples. Even at a relative strain $\varepsilon=0.7 \%$ (Fig. 6 b), fracture was already initiated, however, it was localized in contact zones.

The fracture of the struts of the upper and lower rows of cells occurred at a relative reduction of $3.5 \%$ (Fig. 6 c). Further deformation at $\varepsilon=9.2 \%$ led to the spalling of the material within the upper and lower rows of cells. This process continues at $\varepsilon=14.8 \%$, but the central region of the sample remains intact. Its fracture is observed only at $\varepsilon=27.1 \%$.

Thus, the fracture of the sample occurs stepwise with the transition from the proximity rows to the central ones. This can be explained with the fact that frictional stress is applied on the metal near the contact surface, which, according to the principle of equivalent stresses calculation, must be added to the general stress value following the certain rules of material resistance. The procedure of such an experiment is described in [20] in more detail. A similar type of cellular structure failure was observed in [21]. 
Comparative analysis of the experimental results with the simulation data given in the first part of the study shows the following. The fracture is initially localized in the places where the greatest strains are accumulated and the maximum equivalent stresses occur, i.e. in vertical struts between the cells. In the second stage, the fracture moves into the region of horizontal struts. In the third stage, the failure process passes to a set of struts with a complete spalling of the material upon deformation.

The stress-strain curve is shown in Fig. 7. When the stress plateau responsible for struts bending considered, then the local extrema are observed in this interval.

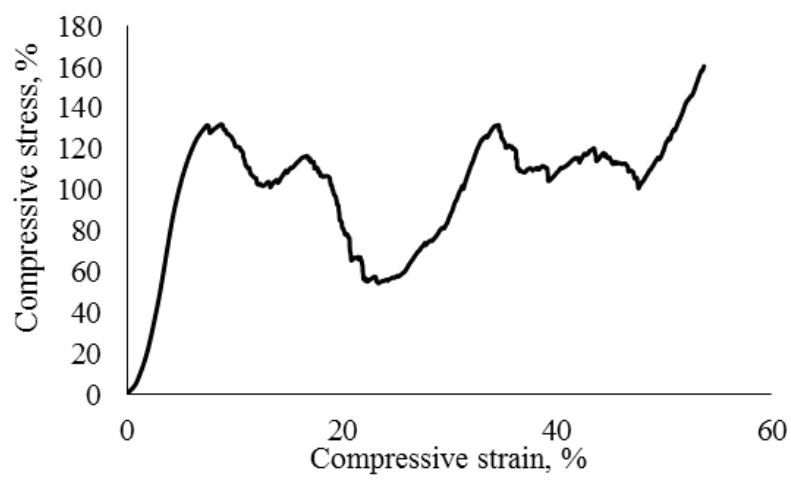

Fig. 7. Compression stress-strain curve for porous implant of titanium alloy

Moreover, one of the minima turns out to be especially deep (at a residual reduction of $21 \%$ ). Two neighboring rows (4 and 5) were destroyed at once during this time interval as can be seen from Fig. 6. The increase in load at the end of the test is associated with the end of the fracture of the struts between the cells. The fractured material densified and formed its own cross-sectional area, as a result, greater stresses are required to transfer the material to the plastic state.

\section{Conclusions}

The boundary conditions were set and the boundaryvalue problem for the titanium implant loading with the cellular architecture was solved using FEA. Data on the stress and strain distribution were obtained. The struts between the individual cells were shown to be dangerous from the point of fracture. To obtain implants with a higher loading capacity, the thickness of the struts should be increased, however, excluding the high values of the modulus of elasticity. The sample of a porous titanium alloy intended for the manufacturing of implants was tested in compression. It was found that the change in cellular structure of the implant under loading occurred stepwise. At first, the peripheral contact regions of the metal were involved in deformation and subsequent fracture. Onward, the deformation extends into the material. The change of cell profile was nonmonotonic during the test due to the localization of stresses and strains within the vertical struts of the cellular structure of the implant. The noted nonmonotonicity led to inhomogenous deformation behavior, which was accomponied with the local extrema in the stress-strain curve.

We hereby acknowledge the support of the Ministry of Science and Education of the Russian Federation, in accordance to the decree of the Government of April 9, 2010, №218, project number 03.G25.31.0234.

\section{References}

1. X. Wang, S. et al., Biomaterials, 83, 127-141 (2016)

2. F.S.L. et al., Acta Biomaterialia, 53, 572-584 (2017)

3. R. Wauthle et al., Acta Biomater, 14, 217-225 (2015)

4. K. Moiduddin et al., Biocybernetics and biomedical engineering, 36, 719-730 (2016)

5. A. Yánez, A. Herrera, O. Martel, D. Monopoli, H. Afonso, Mater. Sci. and Eng.: C., 68, 445-448 (2016)

6. G. Ryan, A. Pandit, DP Apatsidis. Biomaterials 27: 2651-70 (2006)

7. D. Herzog, V. Seyda, E. Wycisk, C. Emmelmann. Acta Materialia, 117, 371-392 (2016)

8. S. Zhang, C. Li, W. Hou, S. Zhao, S. Li. J. of Mat. Sci. \& Tech., 32, 1098-1104 (2016)

9. L. Thijs, F. Verhaeghe, T. Craeghs, J. V. Humbeeck, J.-P. Kruth. Acta Mat., 58, 3303-3312 (2010)

10. F. Wang, S. Williams, P. Colegrove, A. A. Antonysamy., Met. and Mat. Trans.: A., 44A, 968 (2013)

11. B. Piotrowski, A.A. Baptista, E. Patoor, P. Bravetti, A. Eberhardt, P. Laheurte. Mat. Sci. and Eng. C., 38, 151-160 (2014)

12. M. Niinomi, L. Yi, H. Liu, H. Li. Regenerative Biomat., 1-13 (2016)

13. H. Shen, H. Li, L.C. Brinson. Mechanics of Materials, 40, 708-720 (2008)

14. L.J. Gibson, M.F. Ashby. Cellular Solids: Structure and Properties II ed., Cambridge University Press (1997)

15. Yu.N. Loginov. Izvestiya Vysshikh Uchebnykh Zavedenij. Chernaya Metallurgiya., 11, 24-28 (2002)

16. B.I. Kamenetskij, A.L. Sokolov, Yu.N. Loginov. Kuznechno-Shtampovochnoe Proizvodstvo., 6, 21-23 (1996)

17. A.A. Emel'yanov, I.P. Konakova, Yu.N. Loginov, A.A. Popov. Izvestia Akademii nauk SSSR. Metally., 1, 133-137 (1995)

18. A.A. Ershov, Y.N. Loginov, S.L. Demakov., Metallurgist., 8, 659-663 (2015)

19. Yu.N. Loginov. Steel in Translation., 31, 63-68 (2001)

20. Yu.N. Loginov, A.A. Popov, S.I. Stepanov, E.Yu. Kovalev., Titan., 2 (2017)

21. A. Öchsner, K. Lamprecht., Mech. Research Com., 30, 573-579 (2003) 